\title{
Vaccines, antimalarials and recrudescence
}

\section{Opinion}

It has long been believed that latency was only present in Plasmodium vivax or Plasmodium ovale due to hypnozoites, exoerythrocytic forms in the liver. The prevailing opinion until the middle of the last century was that the maximum duration of Plasmodium falciparum infections was less than 2 years. But asymptomatic carriers are common in endemic areas and the transfer of the parasites to nonimmune patients by blood transfusion is common knowledge. And in many cases the donors have been absent from countries with exposure to malaria for durations longer than 2 years. ${ }^{1}$ Already in 1931 it was found in a cohort of 71 schoolchildren Puerto Rico that some Plasmodium falciparum infections continued for up to 121 weeks. ${ }^{2}$ More recent cases of latency, dormancy and recrudescence accumulate. Recrudescence can occur with waning of immunity following departure from endemic areas. Pregnancy, particularly the primigravid state, is a risk factor for severe infection. ${ }^{3-6}$ In mice treated with artesunate recrudescence is related to dephosphorylation. ${ }^{7}$

Treatment failures and first signs of resistance to artemisinin soon become evident already evident in China with arteannuin $B$ and artemisinin monotherapy in 1973. This was due to a novel effect: a small fraction of the parasites, as a result of chemotherapeutic pressure, become 'dormant'. At the ring stage, the parasite cycle is halted, making the parasites unsusceptible to further dosing until wakening. ${ }^{8}$ The parasite encapsulates itself against the aggressive peroxide artesunate and reawakens at the end of the treatment. The same effect is called quiescence by a French research team. ${ }^{9}$ The dormancy effect is also evident for artemisone, a new artemisinin derivative. ${ }^{10}$ The resistance to ACTs leads to the survival of most fit populations of parasites which in turn lead to more virulent infections. ${ }^{11}$ Severe recrudescence has also been noticed in the use of dihydroartemisininpiperaquine and a large increase in the prevalence of parasitemia. Not only the load of asexual parasites increased after 4 months but also the load of gametocytes. ${ }^{12}$

A recent paper rings an alarm bell. Plasmodium chabaudi malaria parasites through a step-wise increase in artesunate dose evolve extremely rapidly slow clearance rates. These slower clearance rates provide fitness advantages to the parasite through increased overall density, recrudescence after treatment and increased transmission potential. Removal of only the susceptible parasites by artesunate treatment led to substantial increases in the densities of resistant parasites. ${ }^{13}$ Several recent studies show dramatic failure rates for sulfadoxine-pyrimethamine-IPT. In Malawi a recrudescence of $33 \%$ after PCR correction was noticed. $95 \%$ of the women with asymptomatic parasitaemia carried a quintuple mutant and the survival rate of the malaria infected primigravidae remained disastrous despite the treatment. Since many years it is well known that pyrimethaminesulfadoxine (SP) even increases the gametocyte density. In a trial in South Africa the duration of gametocyte carriage increased from 3 to 22 weeks between 1998 and 2002. ${ }^{14,15}$ Folate supplementation in pregnancy is universally recommended as part of antenate care. Although international guidelines recommend 0.4 or $0.6 \mathrm{mg}$ daily, many countries in Sub-Saharan Africa use $5 \mathrm{mg}$ daily, because the $5 \mathrm{mg}$ tablet is more widely available. Several studies have shown that folate can antagonize the antimalarial activity of sulfadoxinepyrimethamine in vitro and in vivo. ${ }^{16}$

For chloroquine the situation is even worse. A 5-fold increase in gametytogenesis in Plasmodium falciparum has been documented. ${ }^{17}$ The situation is as dramatic for the RTS, S vaccine which protects only against sporozoites but does not induce clinical immunity against
Volume 6 Issue 2 - 2018

\author{
Pierre Lutgen \\ 'FBV-BELHERB, Luxembourg
}

Correspondence: Pierre Lutgen, IFBV-BELHERB, BP 98 L-6905, Niederanven, Luxembourg, Email lutgenp@gms.lu

Received: February 20, 2018 | Published: March 07, 2018

blood-stage parasites. The vaccine showed evidence of $35,9 \%$ efficacy in the first year after vaccination, but efficacy fell to $2.5 \%$ in the fourth year. The cohort with a high exposure index even showed a negative efficacy during the fifth year (Figure 1). ${ }^{18}$ Even if some people applaud for the $35.9 \%$ protection provided by this vaccine in the first year, it appears meaningless if one compares with the natural healing rate. A longitudinal study was conducted involving 273 children 1-10 years of age with acute, uncomplicated malaria in Kampala, Uganda. Malaria parasitemia was measured at enrollment, on day 3 , and on day 7. Malaria parasitemia had completely cleared in $57.1 \%$ and $85.3 \%$ of children by day 3 and day 7 , respectively. ${ }^{19}$ The effect of 8 antimalarial drugs on gametocyte production was studied in vitro. Exposure to antimalarial drugs resulted in an increase in the number of gametocytes in test cultures. None of the drugs tested statistically significantly reduced gametocyte numbers. ${ }^{20}$ The situation appears to be disastrous for mefloquine monotherapy. Patients treated with mefloquine had a high risk for mosquito infectivity and transmissibility. A disaster is pending for Africa. Lariam is now forbidden in several European countries and remaining stocks are sold in Africa. ${ }^{21}$

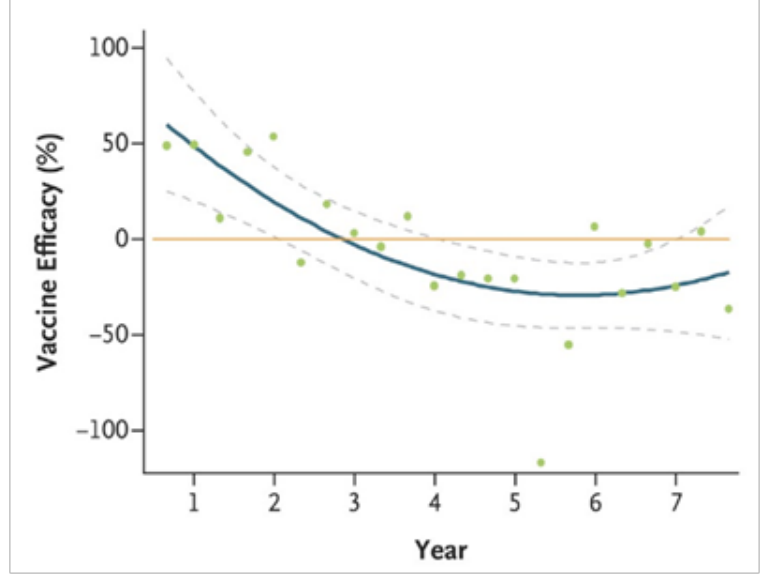

Figure I 5-fold increase in gametytogenesis in Plasmodium falciparum.

Concerning mass drug administration, recently in a paper sponsored by Bill Gates, the Global Fund and WHO the following conclusion is reached : Mass drug administration has the potential to reduce transmission for a limited time, but is not an effective replacement for existing vector control. Unless elimination is achieved, mass drug administration has to be repeated regularly for sustained effect. ${ }^{22}$ Very strange is the effect noticed in several papers 
or reports. Introducing LLINs (long lasting insecticide treated nets) in areas where ACTs are in broad use provokes a sharp jump in malaria incidence. ${ }^{23,24}$ Hopefully a study from the Worcester Technical Institute shows that Artemisia annua dried leaves are able to reverse the resistance induced by artemisinin and derivatives. ${ }^{25}$ In a recent clinical trial in RDCongo, treating uncomplicated malaria with either Artemisia annua or Artemisia afra was superior to the artesunate ACT treatment. At D14-28 gametocyte carriage was undetectable in Artemisia-treated patients, so transmission to the mosquito should be interrupted. ${ }^{26}$ This is an African breakthrough. So far, WHO protocols only have addressed the decrease in parasite density of asexual forms. One may wonder why gametocydal action and transmission blocking potential were not a major issue for Bigpharma WHO ? Cui bono ?

\section{Acknowledgement}

None.

\section{Conflict of interest}

None.

\section{References}

1. Ashley EA, White NJ. The duration of Plasmodium falciparum infections. Malar J. 2014;13:500.

2. Earle WC. The course of naturally acquired malaria. World Health Organisation: Swizterland; 1962. p.53.

3. Al Hammadi A, Mitchell M, Abraham GM, et al. Recrudescence of Plasmodium falciparum in a Primigravida After Nearly 3 Years of Latency. Am J Trop Med Hyg. 2017;96(3):642-644.

4. Kutsuna S, Kobayashi T, Kato Y, et al. Malaria cases treated with artemether/lumefantrine in Japanese travelers. Kansenshogaku Zasshi. 2014;88(6):833-839.

5. Dierksen J, Al-Ibraheemi A, Wanger A, et al. Plasmodium Falciparum Recurrence Two Years after Exposure in Endemic Country: A Case Report. Ann Clin Lab Sci. 2016;46(4):433-434.

6. Berrevoets MA, Sprong T, Meis JF, et al. Plasmodium falciparum malaria recrudescence occurring 2.5 years after leaving an endemic country. Neth J Med. 2013;71(8):426-428.

7. Du Toit A. Parasite biology: Keeping Plasmodium awake. Nat Rev Microbiol. 2018;16(2):63.

8. Andrea C, Franka T, Dennis EK, et al. Artemisinin-induced parasite dormancy: a plausible mechanism for treatment failure. Malar $J$. 2011;10:56.

9. Witkowski B, Lelievre J, Barragan MJ, et al. Increased tolerance to artemisinin in Plasmodium falciparum is mediated by a quiescence mechanism. Antimicrob Agents Chemother. 2010;54(5):1872-1877.

10. Grobler L, Chavchich M, Haynes R. Assessment of the induction of dormant ring stages in Plasmodium falciparum parasites by artemisone. Antimicrob Agents Chemother. 2014;58(12):7579-7582.

11. Hott A. Altered erythrocytic development phenotypes of artemisinin resistant Plasmodium falciparum confer a fitness advantage. University of South Florida: USA; 2014. pp.181.
12. Coldiron ME, Grais RF. Intermittent preventive treatment for malaria among children in a refugee camp in Northern Uganda: lessons learned. Malar J. 2017;16:218.

13. Pollitt LC, Huijben S, Sim DG, et al. Rapid Response to Selection, Competitive Release and Increased Transmission Potential of Artesunate-Selected Plasmodium chabaudi Malaria Parasites. PLoS Pathog. 2014;10(4):e1004019.

14. Julie G, Dyson M, Ryan EW, et al. In vivo efficacy of sulphadoxinepyrimethamine for the treatment of asymptomatic parasitaemia in pregnant women in Machinga District, Malawi. Malar $J$. 2015;14:197

15. Karen IB, Francesca L, Aaron M, et al. Increased Gametocytemia after Treatment: An Early Parasitological Indicator of Emerging Sulfadoxine-Pyrimethamine Resistance in Falciparum Malaria. $J$ Infect Dis. 2008;197(11):1605-1613.

16. Ouma P, Parise ME, Hamel MJ, et al. A randomized controlled trial of folate supplementation when treating malaria in pregnancy with sulfadoxine-pyrimethamine. PLoS Clin Trials. 2006;1(6):e28.

17. Buckling A, Miles A, Read AF, et al. Chloroquine increases Plasmodium falciparum gametocytogenesis in vitro. Parasitology. 1999;118(Pt 4): 339-346.

18. Olutu A, Fegan G, Bejon P, et al. Seven-year efficacy of RTS,S/ AS01 malaria vaccine amoung young African children. New England Journal of Medicine. 2016;374:2519-2529.

19. Amy M, Mukasa G, Shankar AH, et al. Antioxidant status and acute malaria in children in Kampala, Uganda. Am J Trop Med Hyg. 2001;65(2):115-119.

20. Peatey CL, Skinner-Adams TS, Dixon MW, et al. Effect of antimalarial drugs on Plasmodium falciparum gametocytes. $J$ Infect Dis. 2009;200(10):1518-1521.

21. Akintunde S, Oluchi ON, Okuboyejo TM, et al. Effects of mefloquine and artesunate mefloquine on the emergence, clearance and sex ratio of Plasmodium falciparum gametocytes in malarious children. Malar J. 2009;8:297.

22. Oliver JB, Hannah CS, Peter PR, et al. Role of mass drug administration in elimination of Plasmodium falciparum malaria: a consensus modelling study. Lancet Glob Health. 2017;5(7):e680e687.

23. Johan U, Lars R, Amabelia R, et al. Malaria Transmission in Bissau, Guinea-Bissau between 1995 and 2012: Malaria Resurgence Did Not Negatively Affect Mortality. PLoS One. 9(7):e101167.

24. World Health Organisation. Mass drug administration for malaria. Switzerland: Malaria Policy Advisory Committee; 2017 Mar. 30p.

25. Mostafa AE, Melissa JT, Nicholas GR, et al. Dried whole-plant Artemisia annua slows evolution of malaria drug resistance and overcomes resistance to artemisinin. Proc Natl Acad Sci. 2015;112(3):821-826.

26. Jerome M, Lucile CV, Michel I, et al. Artemisia annua and Artemisia afra tea infusions were equal to or better than artesunate-amodiaquine (ACT) in treating Plasmodium falciparum malaria in a large scale, double blind, randomized clinical trial. 